\title{
Optical angular momentum conversion in a nanoslit: reply
}

\author{
Philip F. Chimento, ${ }^{1, *}$ Paul F. A. Alkemade, ${ }^{2}$ Gert W. 't Hooft, ${ }^{1,3}$ and Eric R. Eliel ${ }^{1}$ \\ ${ }^{1}$ Huygens Laboratory, Leiden University, P.O. Box 9504, 2300 RA Leiden, The Netherlands \\ ${ }^{2}$ Delft University of Technology, Kavli Institute of Nanoscience, Lorentzweg 1, 2628 CJ Delft, The Netherlands \\ ${ }^{3}$ Philips Research Laboratories, Professor Holstlaan 4, 5656 AA Eindhoven, The Netherlands \\ *Corresponding author: philip.chimento@gmail.com
}

Received July 11, 2013; accepted July 15, 2013;

posted August 28, 2013 (Doc. ID 193613); published September 26, 2013

\begin{abstract}
We respond to a Comment on our Letter [Opt. Lett. 37, 4946 (2012)], in which we reported on the spin-to-orbital optical angular momentum conversion of a circular nanoslit in a thin metal layer. We claimed, in an unfortunately worded sentence, that the conversion efficiency was independent of the slit's dichroism, which the Comment pointed out was incorrect. We acknowledge this and reiterate our original intention that as long as the dichroism is not too large, then it has little effect on the conversion efficiency in our system. ( 2013 Optical Society of America

OCIS codes: (310.6628) Subwavelength structures, nanostructures; (230.7370) Waveguides; (260.1440) Birefringence; (050.1930) Dichroism; (240.6680) Surface plasmons; (050.4865) Optical vortices.

http://dx.doi.org/10.1364/OL.38.003891
\end{abstract}

We reply to Dr. Brasselet's Comment on our Letter, "Optical angular momentum conversion in a nanoslit" [1]. We regretfully acknowledge upon rereading the sentence in question on p. 3 of our manuscript that states "We find that the polarization conversion efficiency $\eta$ is independent of the slit's dichroism," that the text gives the wrong impression.

In hindsight, the text should have read "largely independent," which is more in keeping with our assertion in the paragraph following that the "optical spin-orbit conversion is a universal property of a circular nanoslit as long as the local polarization eigenmodes have... similar damping." Although the Comment is completely correct, we still wish to demonstrate that if the dichroism $\delta$ does not get too far away from unity, the efficiency $\eta$ is still, to a reasonable experimental approximation, equal to its value when $\delta=1$.

Equation (3) in the Comment reduces to

$$
\eta=\frac{1}{2}-\frac{\delta}{1+\delta^{2}} \cos \Delta
$$

where $\Delta$ represents the birefringent phase retardation. This expression is equal to Eq. (2) in our original manuscript, $\eta=\sin ^{2}(\Delta / 2)$, if $\delta=1$. However, if we take $\Delta=\pi$ (a half-wave plate) and $\delta=0.8$ as an example, we get a deviation in $\eta$ of only $1.2 \%$ from its ideal value when $\delta=1$; whereas $\delta$ deviates from its ideal value by $20 \%$. That is to say, the slit design is very forgiving when it comes to variations in the slit's dichroism. This is also apparent in Fig. 1(a) of the Comment, which shows the parameter space of $\eta(\Delta, \delta)$. If one looks only at the region $\delta \gtrsim 0.6$, the value of $\eta$ varies considerably with $\Delta$ along the vertical axis of the plot, whereas it hardly varies at all with the dichroism in the horizontal direction.

\section{Reference}

1. P. F. Chimento, P. F. A. Alkemade, G. W. 't Hooft, and E. R. Eliel, Opt. Lett. 37, 4946 (2012). 